\title{
The Changing Nature of QU Carinae: SN Ia Progenitor or a Hoax?
}

\author{
Stella Kafka \\ NASA Astrobiology Institute and Department of Terrestrial Magnetism, \\ Carnegie Institution of Washington, \\ 5241 Broad Branch Road NW, \\ Washington, DC 20015, USA \\ email: skafka@dtm.ciw.edu
}

\begin{abstract}
The race to the elusive Type Ia supernovae (SNe Ia) progenitors is at its zenith, with numerous clues from SNe Ia ejecta and a dearth of observational candidates. Still, the single degenerate channel is a viable route of mass accumulation onto a white dwarf to the Chandrasekhar limit. I present long-term high resolution spectroscopy of QU Carinae, one of the most promising single degenerate SNe Ia progenitors. I discuss its highly variable nature and compare it to current scenarios for mass accumulation onto high-mass white dwarfs, eventually leading to WD detonation and to a supernova explosion.
\end{abstract}

Keywords. binaries: general

\section{Introduction}

In a conference where Type Ia supernovae Ia (SNe Ia) progenitors are the main players, an extended introduction on the proposed channels leading to an explosion is redundant. If not anything else, we all agree that these progenitors are binaries with at least one white dwarf (WD) component and a white dwarf (double degenerate path) or main sequence/giant star (single degenerate path) companion. At the same time and despite the numerous debates on the nature of the companion, models of the progenitors as well as observations of the remnants and of explosion delay times have provided strong indications on what that progenitor may be. Today, I will discuss an object that belongs in the category of semi-detached binaries, and I will argue that it is qualified to be considered a possible SN Ia progenitor.

QU Carinae (hereafter QU Car) belongs to the V Sagittae (V Sge) family of binary systems, in which a white dwarf is accreting mass with mass transfer rate $(\dot{M})$ of $\sim 10^{-7} \mathrm{M}_{\odot}$ $\mathrm{yr}^{-1}$ from a companion. The light curves of $\mathrm{V}$ Sge binaries exhibit bright and faint states, which are very well described by the accretion wind evolution (AWE) scenario (Hachisu \& Kato 2003). In short, during the bright phase, mass is transferred onto the WD from the donor star with $\dot{M} \sim 10^{-7} \mathrm{M}_{\odot} \mathrm{yr}^{-1}$. This results in an increase of the disk size from $1.15 \mathrm{R}_{L, W D}$ to $6 \mathrm{R}_{L, W D}$ on dynamical timescales, where $\mathrm{R}_{L, W D}$ is the Roche lobe radius of the white dwarf of the system, and to a slow expansion of the WD's atmosphere. When the photospheric mass reaches $0.07 \mathrm{M}_{\odot}$, a massive wind phase is also initiated; the mass blown in the wind is of the order of $10^{-6} \mathrm{M}_{\odot} \mathrm{yr}^{-1}$. This wind is essentially a free stream with velocities reaching $1,000 \mathrm{~km} \mathrm{~s}^{-1}$ (larger than the orbital period of the binary) and it collides with the donor star, removing its surface layers. The result is for mass transfer from the donor star to be suppressed and subsequently stop, while the disk is drained onto the WD. When the wind phase stops, the white dwarf contracts to its initial value of $0.02 \mathrm{R}_{\odot}$ and the brightness of the system gradually decreases to the faint optical state. The cycle is repeated once mass transfer starts again. 
The wind phase is essential to the mass accumulation of the system, since it prevents a common envelope from forming around the binary as a result of the photospheric expansion of the white dwarf during the bright phase and a merging of the two stars of the binary. The V Sge systems represent a phase of this evolution that should last $\sim 10^{5}$ years. Hachisu \& Kato (2003) predict that about 100 such objects should be present in our galaxy alone at all times; we now know of six candidate sources (including the prototype), and I will discuss the latest addition to the category, which is QU Car.

\section{QU Carinae}

QU Carinae was discovered as a bright galactic source, a possible twin to the low-mass x-ray binary Sco X-1 (Stephenson et al. 1968). Subsequently, optical photometry revealed erratic variations in the short-term light curve of the binary having amplitude of 0.1-0.2 mag on timescales of a few minutes (Gilliland \& Phillips 1982). These were interpreted as being due to the flickering on the bright accretion disk of the system rather than orbital brightness variations.

A period of $10.9 \mathrm{~h}$ for the binary was derived from the optical HeII $4686 \AA$ lines of the binary (Gilliland \& Phillips 1982) but was not confirmed in subsequent optical observations (Kafka et al. 2008). In the optical, all Balmer lines are in emission; the dominated species is the Bowen blend (CIII/NIII/OIII) at 4630-4660 $\AA$ reaching up to two times the strength of the HeII $4686 \AA$ emission line. Using the radial velocity centroid, Gilliland \& Phillips (1982) argue that the CIII triplet lines at $4647-4652 \AA$ are the dominant species in the blend. A carbon overabundance is also suggested by the strong CIV 5801, $5812 \AA$ lines in the optical spectra of the system, which occasionally display P-Cygni profiles, indicating the presence of an outflow reaching $5,700 \mathrm{~km} \mathrm{~s}^{-1}$ (Kafka et al. 2008). In the optical spectra there are no absorption features from the secondary star. Drew et al. (2003) attributed the overabundance of carbon in the optical and UV spectra of the binary to the atmosphere of the secondary star, suggesting that it is an R-type carbon star.

QU Car has both IUE and HST/STIS UV spectra: using the rich suite of interstellar lines in the HST/STIS spectra, Drew et al. (2003) derived a distance of $2 \mathrm{kpc}$ for QU Car. For that distance, the luminosity of the object is estimated at $10^{37} \mathrm{ergs} \mathrm{s}^{-1}$ and the mass transfer rate at $\dot{M} \sim 10^{-7}-10^{-6} \mathrm{M}_{\odot} \mathrm{yr}^{-1}$. The bright HeII $1640 \AA$ emission and the strong OV $1371 \AA$ absorption lines signal a higher than usual nova-like ionization levels in QU Car (indicating a very hot ionizing source). Further signals of an erratic outflow from the system were provided by mass-loss signatures in NV $1240 \AA$ and CIV $1549 \AA$.

The presence of a nebula around the system was supported by Kafka et al. (2008), who found [OIII] $5007 \AA$ and [NII] $6584 \AA$ emission. Higher resolution spectra should, in principle, resolve features of the nebula and pinpoint its origin. I present here two epochs of snapshot echelle observations of the binary, revealing its variable and erratic nature.

\section{QU Car in 2010-2011}

The long-term light curves of the system from ASAS $\dagger$ and AAVSO $\ddagger$ exhibit bright and faint states (top panel of Figure 1 and Kafka et al. 2008), similar to those of the prototype of the class, V Sge. The optical state of the system is derived based on the appearance and properties of the spectral features of the star. Our snapshot high

$\dagger$ All Sky Automated Survey http://www.astrouw.edu.pl/asas/

$\ddagger$ American Association of Variable Star Observers www.aavso.org/ 
resolution ( $\mathrm{R} \sim 30,000)$ data were obtained using the echelle spectrograph on the Du Pont telescope of the Las Campanas observatories (www.lco.cl), during five observing nights in 2010 and 2011. Examples of the two epochs of spectra are presented in Figure 1 (bottom), and are centered around the $\mathrm{H} \alpha$ and $\mathrm{H} \beta$ lines and the Bowen blends (from right to left). The higher resolution of this data set reveals unprecedented structure in the Balmer line profiles, unveiling different components that are not easily distinguished with lower resolution. Both Balmer lines presented here have similar structure to those in $\mathrm{V}$ Sge, with components reaching radial velocities $\pm 550 \mathrm{~km} \mathrm{~s}^{-1}$. At the same time, they both seem to be significantly attenuated in 2010, indicating that the binary may have been transitioning to a faint state in 2010. Lack of a secure ephemeris doesn't allow decomposition of the different line components and their correlation with different parts of the binary. At the same time, there is a blueshifted stationary feature at $-135 \mathrm{~km} \mathrm{~s}^{-1}$ in both Balmer lines (at both epochs of observations), which is not connected kinematically to any other features and seems to be circumbinary in nature. Furthermore, there is a pair of absorption features in the red and blue sides of the $\mathrm{H} \beta$ line. Having

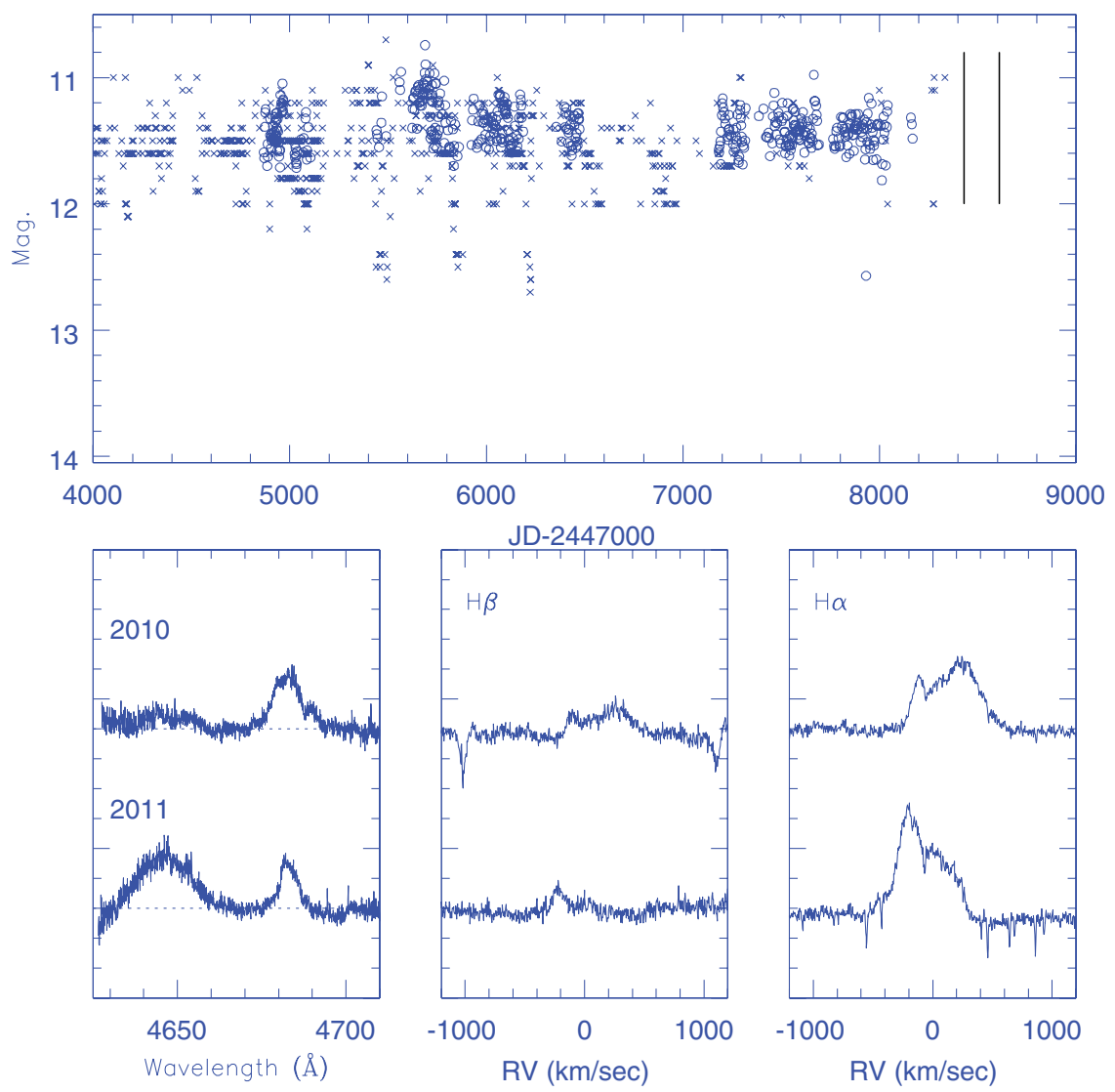

Figure 1. Top: Long-term AAVSO visual (crosses) and ASAS V Magnitude (open circles) light curve of QU Carinae. It demonstrates that over time, the system exhibits V Sge-type bright and faint states, albeit the latter are shorter-lived than those of the prototype. The times of our 2010-2011 spectroscopic observations are also marked in the figure. Bottom: snapshot observations of the main spectral features of QU Car at the two epochs of our observations. From left to right, we present the Bowen blend (CIII/NIII/OII) at 4630-4660§ and HeII $4686 \AA$ (left panel), the $\mathrm{H} \beta$ emission (central panel), and the $\mathrm{H} \alpha$ emission components (right panel). Relevant discussion is in the text. 
excluded artificial contamination in our spectra, the features are convincingly real. The red component is stationary, reaching $1,110 \mathrm{~km} \mathrm{~s}^{-1}$. The red component is present only during two subsequent nights of our observations (in E1 and in E2) at $-370 \mathrm{~km} \mathrm{~s}^{-1}$ and $-1,050 \mathrm{~km} \mathrm{~s}^{-1}$ respectively. They resemble line components commonly encountered in Be stars where they represent outflowing and inflowing material. In QU Car, it is very possible that these absorption line components represent expanding gas in the vicinity of QU Car; however a very specific orientation of this gas would be required to allow for their kinematic behavior.

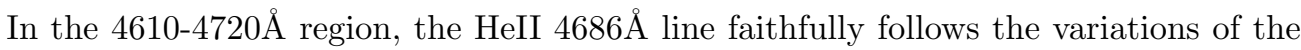
Balmer lines. In the 2010 spectra, the Bowen blend appears significantly attenuated, reaching noise level at times, echoing the behavior of the Balmer lines. At the same time, the CIV 5801, 5812 $\AA$ lines are not present in the system. If the dominant species for this Bowen blend is CIII from the donor star, then we can not explain this variation; however, CIII can also come from a CO-rich white dwarf. According to the accretion wind evolution scenario, the faint state corresponds to a significant depression of the white dwarf's photosphere, which is expanded during the wind phase. Therefore, we can interpret the attenuated Bowen blend in the faint state of QU Car as being the white dwarf's photospheric emission and conclude that QU Car was indeed in a faint state during the 2010 spectroscopic observations.

\section{A Type Ia Supernova Progenitor?}

The main point of our discussions throughout this meeting is to identify possible supernova Ia progenitors and/or quantify their properties. Mohamed's simulations (this volume) present $\mathrm{NaI} \mathrm{D}$ and $\mathrm{CaII} H \& \mathrm{~K}$ circumbinary material from RS Oph, which is considered a possible SN Ia progenitor. Along those lines, Williams (this volume) discussed spectral features that were detected after a nova explosion and are likely related to debris from the donor star of the nova progenitor, which are ejected at quiescent times. From the supernova community, Patat (this volume) and Sternberg (this volume) report variable $\mathrm{NaI} \mathrm{D}$ absorption line components which are observed in SNe Ia, and are related to the progenitor system. The main idea in their interpretation is that NaI atoms are present in the SN Ia progenitor; after the explosion, the atoms are ionized by the UV radiation from the $\mathrm{SN}$ and then slowly recombine over the following weeks to produce the variability in line strengths that are observed. From all the above, it is clear that circumbinary material can provide diagnostics for the identification of SNe Ia progenitors, and can be used to evaluate whether any of the suspected possible channels can lead to an SN Ia explosion.

In QU Car, both the NaI D and CaII H\&K lines are in absorption. However, they seem to be stationary at each epoch of observations, changing very slowly between epochs in radial velocity space, from $-6 \mathrm{~km} \mathrm{~s}^{-1}$ in 2010 to $-13.5 \mathrm{~km} \mathrm{~s}^{-1}$ in 2011 . The lines are not kinematically associated with any other emission or absorption component of the system. A lack of any night-to-night variation is consistent with them being circumbinary in nature, indicating the presence of slowly expanding material similar to the one predicted in hydrodynamic models around high mass-transfer rate CVs (Mohamed, this volume). This can be ejected from a wind and expand slowly from the system, similar to the circumbinary material found after nova explosions from Williams et al. (2008, this volume) and in the debris from a number of SNe Ia in the Patat et al. and Sternberg et al. studies. This circumbinary material can be the missing link, bridging the SNe Ia explosions to their progenitors, making QU Car a very likely SN Ia candidate. 
I guess that the main difficulty in interpreting QU Car data is that one simply cannot use a few snapshot spectra to argue for a particular scenario. The spectrum varies greatly from epoch to epoch, and it is likely that the full range of behaviors is even broader that is being shown here. Nevertheless, QU Car is an interesting object by itself and very likely an SN Ia progenitor in our galaxy.

\section{Acknowledgements}

I would like to acknowledge a NASA Astrobiology Institute postdoctoral fellowship that made this research (and my participation in this meeting) possible.

\section{References}

Drew, J. E., Hartley, L. E., Long, K. S., \& van der Walt, J. 2003, MNRAS, 338, 401

Hachisu, I. \& Kato, M. 2003, ApJ, 598, 527

Gilliland, R. L. \& Phillips, M. M. 1982, ApJ, 261, 617

Kafka, S., Anderson, R., \& Honeycutt, R. K. 2008, AJ, 135, 1649

Stephenson, C. B., Sanduleak, N., \& Schild, R. E. 1968, ApL, 1, 247

Williams, R., Mason, E., Della Valle, M., \& Ederoclite, A. 2008, ApJ, 685, 451 